\title{
PRECISE LOCAL TEMPERATURE MEASUREMENT OF FULLY ENCAPSULATED OVENIZED MEMS DEVICES \\ Hyun-Keun Kwon ${ }^{1 *}$, David B. Heinz ${ }^{1}$, Dongsuk D. Shin ${ }^{l}$, Yunhan Chen ${ }^{2}$, Lizmarie C. Ortiz ${ }^{l}$, Gabrielle D. Vukasin ${ }^{1}$, and Thomas W. Kenny ${ }^{1}$ \\ ${ }^{1}$ Stanford University, California, USA \\ ${ }^{2}$ Apple, Cupertino, California, USA
}

\begin{abstract}
In this paper, a simple and precise method is proposed to non-invasively monitor localized temperatures of fully encapsulated, suspended and ovenized MEMS devices. By placing clamped-clamped (CC) beams at multiple locations within the device layer and measuring the resonant frequencies, an accurate temperature profile of the entire suspended device can be measured. This method has a small foot print that can be incorporated in any ovenized devices and the measured temperatures can be used to improve temperature control schemes. Compared with an IR-optical or direct 4-point resistance measurement, the frequency measurement offers better resolution with minimal impact on the actual device temperature.
\end{abstract}

\section{INTRODUCTION}

Due to small size and compatibility with conventional silicon integrated circuit (IC) design, MEMS oscillators exhibit great potential for use as time references [1,2]. Fully-encapsulated silicon-based oscillators are now available and have begun to displace quartz crystal oscillators [3]. However, MEMS oscillators suffer from an inherit temperature dependence of frequency (TCf) of about $30 \mathrm{ppm} /{ }^{\circ} \mathrm{C}$ [4]. This results in a large frequency variation compared to quartz crystals. In order to overcome this issue, numerous passive compensation schemes have been introduced such as doping [5] and composite materials [6] that can effectively reduce the TCf without applying additional external power.

Besides the aforementioned methods, state-of-the-art frequency stability has been achieved with an active-ovenization scheme, where the device is controlled at a set temperature above the operating temperature to suppress any remaining temperature dependence [7]. In order to achieve extreme stability, an accurate measurement of the resonator body temperature is crucial as it provides the control signal to maintain operation at the target temperature. Previous instruments utilize a dual-mode operation, where one resonator body is driven with two independent vibrational modes. These two modes exhibit a very different temperature dependence and so the frequency difference between the two modes can be utilized as a high-resolution temperature sensor. This approach has been used to achieve a frequency stability of about $\pm 250 \mathrm{ppb}$ from -20 to $80^{\circ} \mathrm{C}$ range [8].

In order to further improve the frequency stability, a more local understanding of the temperature profile of an encapsulated ovenized resonator is required. Any thermal gradient within the resonator body will introduce an error in the temperature measurement and this problem has to be addressed by an improved, locally-precise measurement scheme. Although a resistive temperature detector (RTD) can be fabricated within this MEMS process, RTD based systems suffer from separation from the resonator and self-heating and are not ideal candidates to monitor localized temperatures [9]. In this paper, we present a frequency-based small foot print temperature measurement method for ovenized MEMS device applications.

\section{DESIGN AND FABRICATION}

A bulk-type resonator with device layer heater is designed and fabricated for this study. Figure 1 (a) shows the overall diagram of the fabricated device. The entire device is suspended by two anchor points where the bias and heating voltages are applied. The main device is a $400 \mu \mathrm{m}$ X $400 \mu \mathrm{m}$ X $40 \mu \mathrm{m}$ Lamé-mode resonator suspended from a frame, which is suspended from the anchors. The suspension frame not only serves as a mechanical support for the resonator body but also provides separation from a temperature gradient present at the heaters. Within the suspension frame, $8 \mathrm{CC}$ beams are included with sense/drive electrodes for capacitive measurement as shown in Figure 1 (b) with dimensions of $190 \mu \mathrm{m} \mathrm{X}$ $3.23 \mu \mathrm{m} \mathrm{X} 40 \mu \mathrm{m}$. To ovenize the device, a DC current flows between through the suspension structures, acting to raise the temperature of the frame via Joule heating. The folded-beam design of the heater provides a large thermal resistance between the device and the ambient to allow a large temperature increase.

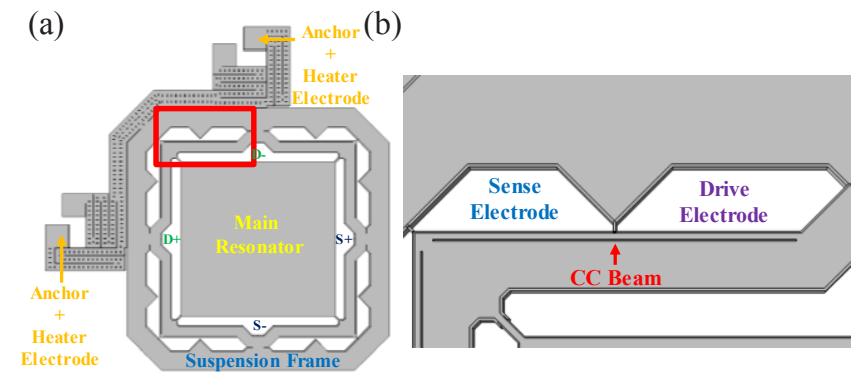

Figure 1. (a) Diagram of bulk-type resonator device layer with an in-plane heater suspending the entire frame. (b) Detailed look into the CC beams within the suspension frame.

The device was fabricated using a wafer-level encapsulation process (epi-seal) co-developed by Robert Bosch Research and Technology Center and Stanford University. The device is fabricated on a (100) N-Type (Phosphorous) SOI wafer with a 40 $\mu \mathrm{m}$ single-crystal silicon device layer, aligned with $<110>$ crystal orientation and doping level of $\sim 2.3 \mathrm{e} 19 \mathrm{~cm}^{-3}$. The doping level plays a critical role in determining the TCf characteristics of silicon resonators [5] and this will be examined more closely in the following section. A variation of this process allows fabrication of etch-hole free bulk-type resonators with high quality factors and seals the device inside a low-pressure cavity $(<\sim 1 \mathrm{~Pa})$ without any moisture, oxygen and other contaminations [9]. By placing the heater within the device layer, a low-power operation is enabled by minimizing heat loss via conduction or convention from the resonator to the rest of the die [7]. Figure 2 shows the top-view SEM image of fabricated device. 


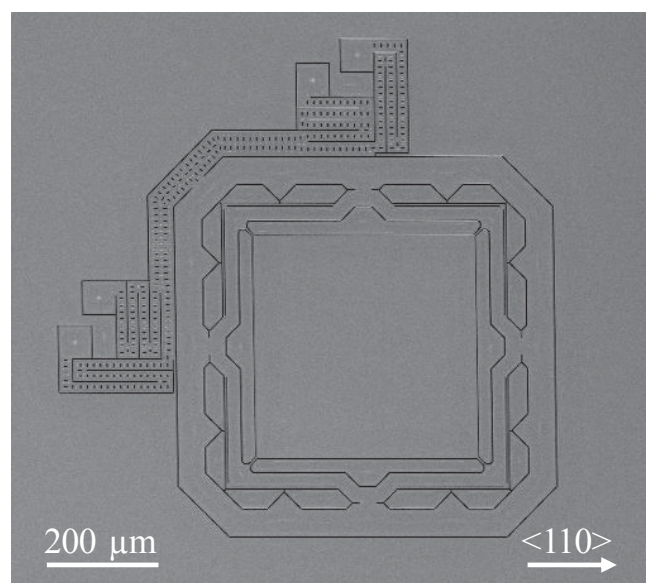

Figure 2. Top-view SEM image of the fabricated device. Scale bar of $200 \mu \mathrm{m}$. The arrow indicates $<110>$ direction of the single-crystal silicon orientation.

\section{EXPERIMENT CHARACTERIZATION}

All the experiments were carried out in a temperature-controlled oven. Operation of the device was done by applying a DC bias to the resonator structure via the heater electrodes and $\mathrm{AC}$ drive voltages were applied to the drive electrodes. The motional current was amplified via a PCB mounted trans-impedance amplifier and measured with a Zurich HF2LI-PLL. As for the Lamé-mode, the device is driven differentially through a 180-Degree phase splitter and sensed through an instrumentation amplifier. Only the MEMS device was placed in the oven; the rest of the system operated in the lab environment. Figure 3 shows the block diagram of the experimental setup.

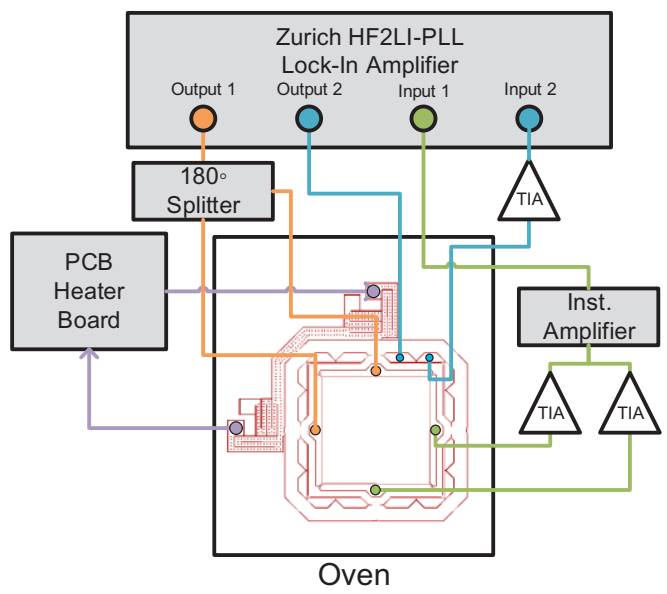

Figure 3. Block diagram of the experimental setup. Both the Lamé mode and CC beam flexural mode can be driven/sensed for frequency measurement.

\section{Open-loop Electrostatic Frequency Measurement}

The open-loop frequency sweeps at $25{ }^{\circ} \mathrm{C}$ show resonant frequencies of $10.34 \mathrm{Mhz}$ for the Lamé-mode and $630.3 \mathrm{kHz}$ for the $\mathrm{CC}$ beam flexural mode. Respective mode-shapes and frequency response are shown in Figure 4 . The $\mathrm{CC}$ beams exhibited mechanical coupling with other $\mathrm{CC}$ beams within the suspension frame and therefore required application of a small bias voltage at each sense or drive electrodes of other $\mathrm{CC}$ beams in order to isolate the single $\mathrm{CC}$ beam of interest. Application of the isolation voltage did not impact the operation of the Lamé-mode. (a)

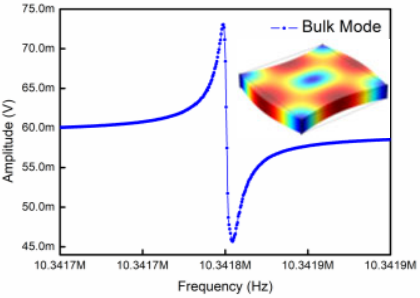

(b)

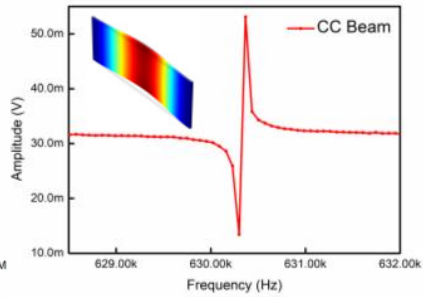

Figure 4. (a) The resonant frequency of the Lamé-mode at $25^{\circ} \mathrm{C}$ temperature. Insert shows the COMSOL simulation of the mode shape. (b) the resonant frequency of the CC beam flexural mode.

For the temperature measurement, we first ran a temperature-sweep of the Lamé-mode and $4 \mathrm{CC}$ beams. 4 beams were chosen such that 2 are closer to the heater and 2 are further apart to investigate the temperature gradient within the device layer (shown in Figure 5 (a)). The resonant peaks were tracked from -20 to $80{ }^{\circ} \mathrm{C}$ with $5^{\circ} \mathrm{C}$ steps and the frequencies were recorded as shown in Figure 5 (b) and (c). As for the Lame-mode, the temperature sweep resulted in a TCf of $-317 \mathrm{~Hz} /{ }^{\circ} \mathrm{C}$ and the 4 beams were about $-16 \mathrm{~Hz} /{ }^{\circ} \mathrm{C}$. Despite the identical design, $4 \mathrm{CC}$ beams exhibited slightly different resonant frequency and temperature dependence due to fabrication variations.

(a)

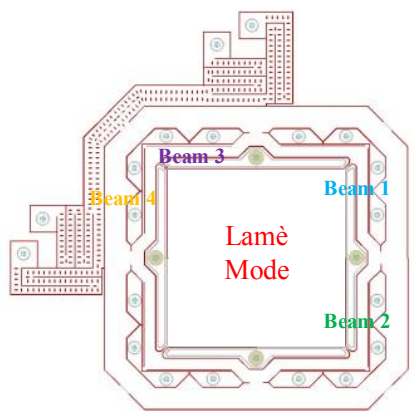

(b)

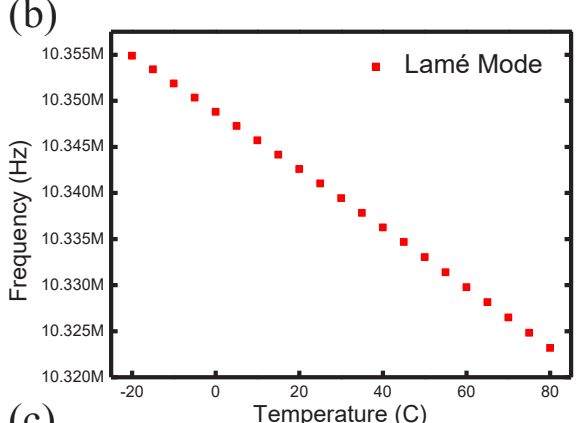

(c)

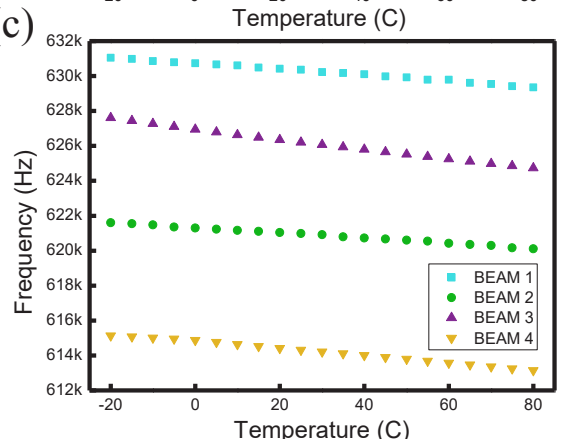

Figure 5. (a) Diagram of the tested device with measured beams labeled. (b) Temperature sweep of the Lamé-mode and (c) 4 beams measured from $-20{ }^{\circ} \mathrm{C}$ to $80{ }^{\circ} \mathrm{C}$ in a temperature-controlled oven. Nonidentical characteristics are shown from the fabricated beams. 
These unique TCf curves are fitted with a $2^{\text {nd }}$-order polynomial and were used in the subsequent test to back-track the temperature of the ovenized device. The main advantage of this method is that the TCf characteristics of these resonators are very repeatable and fairly insensitive to external environmental factors. The incorporated CC beams are very compact and can be tailored to fit in almost any bulk-type devices. For places where in-plane electrodes are not applicable, out-of-plane electrodes can be utilized to sense/drive the CC beams. Moreover, the same setup for measuring frequency can be applied without any additional circuitry thus making this approach very simple to implement.

\section{Ovenization Temperature Results}

To study the temperature profile during ovenized operation, varying heating voltages were applied to the heater electrodes and observed the open-loop frequency sweep. All of the testing was conducted with the oven set at $-20{ }^{\circ} \mathrm{C} \pm 1{ }^{\circ} \mathrm{C}$. As anticipated, the resonator increased in temperature as the heater voltage increased as shown in Figure 6.

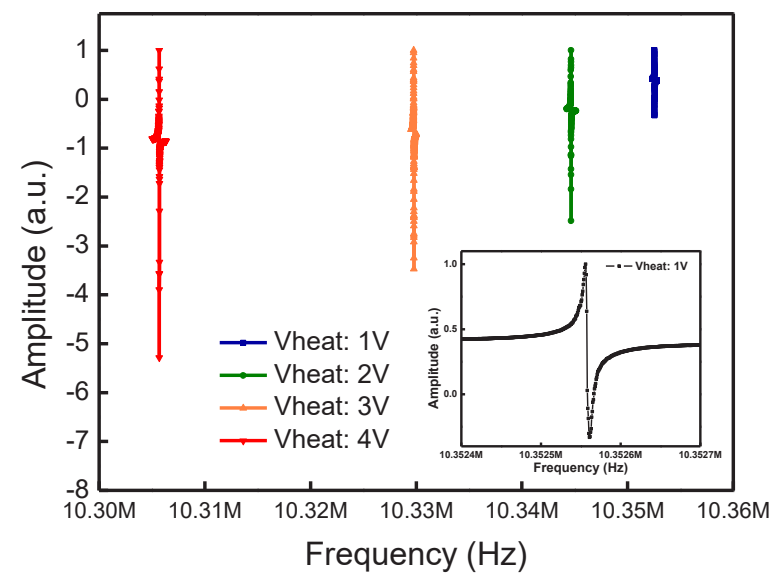

Figure 6. Open-loop sweep of the Lamé-mode with varying heater voltage. From this data and comparing with the fitted TCf curves, temperature of the structure as well as the localized areas of the CC beams can be measured. Peak amplitude normalized to 1. Inset shows a magnified look into the resonant peak at $1 \mathrm{~V}$ heater voltage.

Table 1. Local temperature measurement results within an encapsulated, suspended and ovenized MEMS resonator.

\begin{tabular}{|c|c|c|c|c|c|}
\hline \multirow{2}{*}{$\begin{array}{c}\text { Applied } \\
\text { Heater } \\
\text { Voltage }\end{array}$} & \multicolumn{5}{|c|}{ Temperature $\left({ }^{\circ} \mathbf{C}\right)$} \\
\cline { 2 - 6 } & Lamé-Mode between the Lamé and the beams) & Beam 1 & Beam 2 & Beam 3 & Beam 4 \\
\hline $1.0 \mathrm{~V}$ & -11.8108 & $\begin{array}{c}-12.7447 \\
(-0.9339)\end{array}$ & $\begin{array}{c}-12.1221 \\
(-0.3113)\end{array}$ & $\begin{array}{c}-11.9215 \\
(-0.1107)\end{array}$ & $\begin{array}{c}-11.8895 \\
(-0.0787)\end{array}$ \\
\hline $2.0 \mathrm{~V}$ & 13.1074 & $\begin{array}{c}12.9239 \\
(-0.1838)\end{array}$ & $\begin{array}{c}12.8261 \\
(-0.2813)\end{array}$ & $\begin{array}{c}13.4393 \\
(+0.3319)\end{array}$ & $\begin{array}{c}13.6460 \\
(+0.5386)\end{array}$ \\
\hline $3.0 \mathrm{~V}$ & 59.7742 & $\begin{array}{c}58.9421 \\
(-0.8321)\end{array}$ & $\begin{array}{c}58.9121 \\
(-0.8621)\end{array}$ & $\begin{array}{c}60.5002 \\
(+0.7260)\end{array}$ & $\begin{array}{c}60.7070 \\
(+0.9328)\end{array}$ \\
\hline $4.0 \mathrm{~V}$ & 135.8975 & $\begin{array}{c}135.2130 \\
(-0.6845)\end{array}$ & $\begin{array}{c}135.3251 \\
(-0.5724)\end{array}$ & $\begin{array}{c}136.3546 \\
(+0.4571)\end{array}$ & $\begin{array}{c}136.5121 \\
(+0.6326)\end{array}$ \\
\hline
\end{tabular}

\section{DISCUSSION}

Table 1 shows the result of the frequency-based temperature measurement. For each applied heater voltage, frequencies were measured and the temperatures were back-calculated from the previously mentioned $2^{\text {nd }}$-order polynomial fitted TCf curves. Also, the difference $(\Delta \mathrm{T})$ between the Lamé-mode and each beam is shown.

From these results, it is evident that the temperature profile of the entire device is not uniform and shows that the beams positioned closer to the heater are indeed somewhat warmer than the beams positioned on the opposite side of the frame. Moreover, the Lamé-mode sits at about the middle point between the two ends of the frame. Up to $1.76{ }^{\circ} \mathrm{C}$ temperature difference between the beams were measured at $3.0 \mathrm{~V}$ heater voltage. This non-uniformity can be caused by radiation heat lass throughout the device as well as convective heat loss. These results provide an improvement in understanding of the device temperature profile when incorporated with an in-plane heater design with bulk-mode operation.

To further investigate, a COMSOL simulation with $4.0 \mathrm{~V}$ heater voltage across the micro-oven was conducted with the results shown in Figure 7. The simulation reveals the hot spots located at the folded-beam heaters that are beside the suspension frame. It can be inferred that although the suspension frame assists in uniform heating, the hot spots near the frame and thermal radiation leads to a slight temperature gradient as observed from the experimental results. These results were also observed in previous ovenization devices [11].
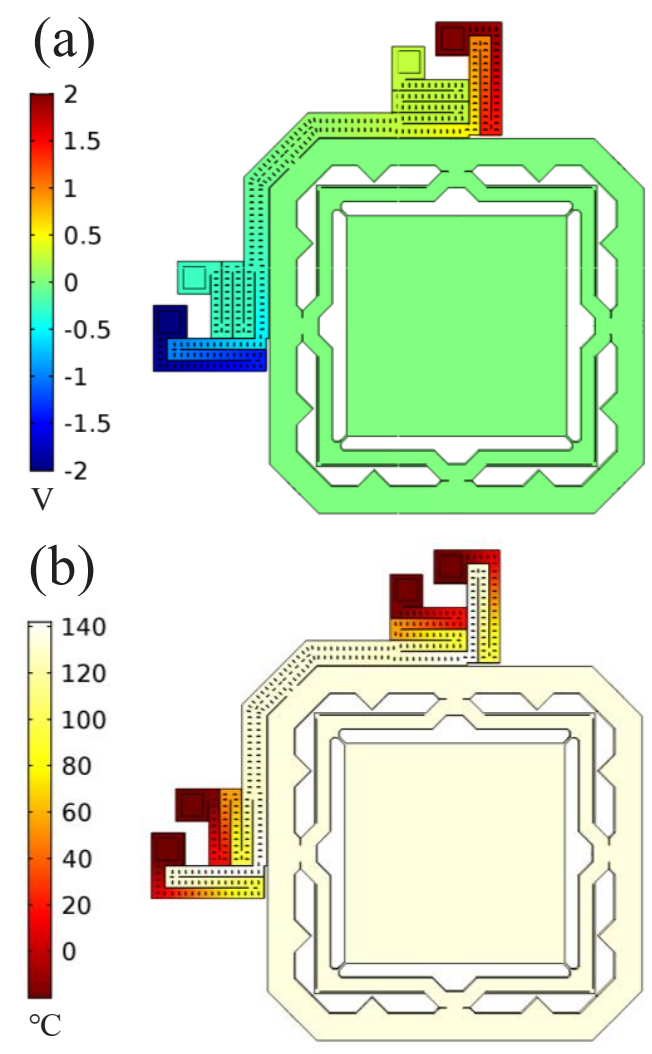

Figure 7. (a) Electric potential profile of the in-plane ovenized device. (b) Temperature profile of the device with $4.0 \mathrm{~V}$ heater voltage applied. This reveals the hotspots near the folded-beam micro-ovens. Electrical conductivity was derived from the doping of the device wafer. 
This method, however, is not without any fault. Since the TCf curve was fitted with a $2^{\text {nd }}$-order polynomial, it cannot guarantee an absolute measurement. Moreover, the accuracy drops for temperatures beyond the temperature sweep points. In addition, any small fluctuations within the oven during the calibration can lead to errors in controlling the frame temperature. In this particular test, the oven was set to each temperature for about 30 minutes to allow settling to the new temperature and smoothing out of any initial temperature gradients.

Despite these non-idealities, these results provide a good understanding of how the in-plane suspended devices can perform under ovenization. For example, this data can be used to provide an additional correction/compensation signal to the heater control feedback loop. Also, for this particular device, a dual-heater with heaters on both sides of the suspension frame should provide a more uniform temperature profile.

\section{CONCLUSION}

In this paper, we have investigated a compact frequency-based temperature measurement scheme that can accurately monitor the temperature profile of an ovenized MEMS device. Compared to optical or RTD based measurements, the frequency-based temperature sensing offers a non-invasive method with no additional circuitry. From the in-plane ovenized device, we observed a temperature gradient of up to $1.76{ }^{\circ} \mathrm{C}$ Additionally, this data has the potential to improve future ovenized device designs. In our future work, we will focus on different heater designs, frame designs and how these design parameters impact the formation of the temperature profile within the device layer to improve the temperature stability of ovenized MEMS resonators.

\section{REFERENCES}

[1] C. Nguyen, "MEMS Technology for Timing and Frequency Control", IEEE Trans. Ultrasonics, Ferroelectrics and Frequency Control, 54, 2, (2007) pp. 251-270.

[2] J.T.M van Beek and R. Puers, "A review of MEMS oscillators for frequency reference and timing applications", J. Micromech. Microeng, 22, 1 (2011)

[3] M. Lutz, A. Partridge, P. Gupta, N. Buchan, E. Klaassen, J. McDonald, and K. Petersen, "MEMS oscillators for high volume commercial applications", in Proc. TRANSDUCERS, Lyon, France, 6/10-14 (2007) pp. 49-52.

[4] R. Melamud, S. A. Chandorkar, B. Kim, H. K. Lee, J. C. Salvia, G. Bahl, M. A. Hopcroft and T. W. Kenny, "TemperatureInsensitive composite micromechanicalresonators", J. Microelectromech. Syst., 18, 6, (2009).

[5] A. Jaakkola, M. Prunnila, T. Pensala, J. Dekker, and P. Pekko, "Determination of doping and temperature-dependent elastic constants of degenerately doped silicon from MEMS resonators", IEEE UFFC, 61, 7, (2014).

[6] V. A. Thakar, Z. Wu, A. Peczalski, M. Rais-Zadeh, "Piezoelectrically Transduced Temperature-Compensated Flexural-Mode Silicon Resonators", J. Microelectromech. Syst., 22, 3, (2013).

[7] J. C. Salvia, R. Melamud, S. A. Chandorkar, S. F. Lord, and T. W. Kenny, "Real-Time Temperature Compensation of MEMS Oscillators Using an Integrated Micro-Oven and a Phase-Locked Loop", J. Microelectromech. Syst., 19, 1, (2010).
[8] Y. Chen, E. J. Ng, D. D. Shin, C. H. Ahn, Y. Yang, I. B. Flader, V. A. Hong, and T. W. Kenny, "Ovenized dual-mode clock (ODMC) based on highly doped single crystal silicon resonators," in Proc. IEEE MEMS, Shanghai, China, 1/24-28 (2016) pp. 91-94

[9] Z. Wu, A. Peczalski, and M. Rais-Zadeh, "Low-power ovenization of fused silica resonators for temperature-stable oscillators," in Proc. IEEE IFCS, Taipei, Taiwan 5/19-22 (2014) pp. 1-5

[10] E. J. Ng, Y. Yang, Y. Chen, and T. W. Kenny, "An etch hole-free process for temperature-compensated, high Q, encapsulated resonators," Solid-State Sensors, Actuators, and Microsystems Workshop, Hilton Head Isl., SC, 6/8-12, Transducer Research Foundation, Cleveland (2014), pp. 99-2014, 99-100

[11] Y. Chen, E. J. Ng, Y. Yang, C. H. Ahn, I. Flader, and T. W. Kenny, "In-Situ ovenization of Lamé-Mode silicon resonators for temperature compensation", in Proc. IEEE MEMS, Estoril, Portugal 1/18-22 (2015) pp. 809-812

\section{ACHKNOWLEDGEMENTS}

This work was supported by the Defense Advanced Research Projects Agency (DARPA) grant "Precise Robust Inertial Guidance for Munitions (PRIGM)", managed by Dr. Ron Polcawich, Contract Number \# N66001-16-1-4023. This work was performed in part at the Stanford Nanofabrication Facility (SNF), supported by the National Science Foundation under Grand ECS- 9731293. Special thanks to all the staffs at SNF for their help during the fabrication process.

\section{CONTACT}

*H.-K. Kwon, tel: +1-650-575-7216; ryankwon@stanford.edu 